\title{
EXPECTATIVAS LABORALES DE ESTUDIANTES UNIVERSITARIOS QUE CURSAN PROGRAMAS EN EL CAMPO SOCIAL
}

\section{EMPLOYMENT EXPECTATIONS OF UNIVERSITY STUDENTS STUDYING PROGRAMS IN THE SOCIAL FIELD}

\author{
Audin Aloiso Gamboa Suárez² \\ Raúl Prada Núñez ${ }^{3}$ \\ César Augusto Hernández Suárez ${ }^{4}$
}

\section{RESUMEN}

Las expectativas laborales se refieren de manera general, a las proyecciones ocupacionales que a mediano o largo plazo tienen los estudiantes

$1 \quad$ El presente artículo hace parte de una investigación avalada por el Grupo de Investigación en Estudios Sociales y Pedagogía para la Paz (GIESPPAZ) con el apoyo de los Semilleros de Investigación en Eduación Matemática, SIRPRILA y SIESPPAZ de la Universidad Francisco de Paula Santander (Colombia)

2 Doctor en Ciencias de la Educación por la Universidad de Cartagena (Colombia). Docente investigador de la Universidad Francisco de Paula Santander. Correo electrónico: audingamboa@ufps.edu.co. Orcid: 0000-00019755-6408.

3 Magíster en Ingeniería de Análisis de Datos, Mejora de Procesos y Toma de Decisiones por la Universidad Politécnica de Valencia (España). Docente investigador de la Universidad Francisco de Paula Santander. Correo electrónico: raulprada@ufps.edu.co. Orcid: 0000-0001-61451786.

$4 \quad$ Magister en Educación Matemática por la Universidad Nacional Experimental del Táchira (Venezuela). Docente investigador de la Universidad Francisco de Paula Santander. Correo electrónico: cesaraugusto@ufps.edu.co. Orcid: 0000-0002-7974-5560. universitarios. De este modo, el presente artículo pretende analizar estas expectativas que tienen los alumnos de un programa de Trabajo Social de una Universidad Pública en Norte de Santander. El enfoque metodológico se inscribe en el paradigma cuantitativo - Descriptivo. Se aplicó una encuesta a 150 estudiantes compuesta de 41 ítems distribuidos en cinco categorías: perfil demográfico, características académicas, situaciones que los informantes valoran en el trabajo, aporte del futuro profesional al ejercicio de su profesión y las características que debería poseer el trabajo ideal. Los resultados demuestran que las expectativas laborales, se pueden relacionar con elementos altruistas, ya que las respuestas de los participantes tienen una tendencia a procurar el bien de las personas de manera desinteresada, incluso a costa del interés propio. 


\section{PALABRAS CLAVE:}

expectativas laborales, educación superior, trabajo social

\begin{abstract}
Job expectations generally refer to the occupational projections that university students have in the medium or long term. In this way, this article aims to analyze these expectations that students of a Social Work program of a Public University in Norte de Santander have. The methodological approach is part of the quantitative - descriptive paradigm. A survey was applied to 150 students composed of 41 items distributed in five categories: demographic profile, academic characteristics, situations that informants value at work, contribution of the future professional to the exercise of their profession and the characteristics that the ideal job should have. The results show that job expectations can be related to altruistic elements, since the responses of the participants have a tendency to disinterestedly seek the good of people, even at the expense of self-interest.
\end{abstract}

\section{KEY WORDS:}

job expectations, higher education, social work

\section{INTRODUCCIÓN}

Cuando nos referimos a expectativas, la Real Academia Española (RAE, s.f) señala que esta se refiere a una esperanza y una posibilidad de que algo se dé posterior a una acción o proceso, parece pertinente también evocar para el presente objetivo investigativo lo que refieren Cabezas, et al. (2013) desde un concepto nominal referente a las expectativas, motivaciones y satisfacción que los estudiantes de cierta carrera tienen en torno a esta, el autor señala que "aquello que se espera antes de empezar la carrera" (p. 17); pero además, por la naturaleza de su documento, Cabezas, et al. nos acerca de paso a lo que son las salidas laborales - "Posibilidad de trabajar en un futuro. Estudiar la carrera para obtener un mayor número de salidas hacia el mercado laboral." ( $p$. 17) - y la posición laboral - "Cargo que se ocupa en una empresa o cualquier actividad laboral. Con mayor nivel académico se obtienen mejores puestos de trabajo." (p.17); temas de interés también en el abordaje del presente problema de investigación.

Hablar de expectativas, además, nos remite al por qué no permanecen los nuevos trabajadores en sus puestos en el sentido de que "El ajuste de las expectativas supone que, en un momento determinado, la realidad ante la que el joven se está enfrentando, no difiere con relación a lo que él esperaba que fuera." (Pinazo y Carrero, 2001, párr. 2); situación que si se torna contraria (desajuste de las expectativas), puede concurrir en "consecuencias negativas para los resultados afectivos y puede llevar a disminuir el compromiso con la organización." (Wanous, 1977, citado en Pinazo y Carrero, 2001, párr. 5). Por lo anterior se hace necesario reconfigurar estos desajustes, aporte que podemos obtener de Paz, et al. (2014) cuando menciona que "la formación profesional debe valorar en qué medida su perfil de egreso permite satisfacer las demandas del mercado laboral, que está sujeto a condiciones precarias de empleo, factores culturales, habilidades mostradas y potencial del factor humano" (p. 67); dichos autores, mencionan la dificultad de los egresados universitarios para ingresar al campo laboral y consecuencias como el ganar autonomía hasta los 40 años o el apartarse de proyectos personales, panorama por el cual

[...]muchos de los jóvenes optan por aceptar ofertas de empleo muy por debajo de su formación profesional, vincularse a trabajos no acordes con su profesión o aceptar empleos precarios que pueden ser temporales, de tiempo parcial, trabajar por su cuenta o en la 
informalidad. Trabajos todos estos que no cumplen con las expectativas de los jóvenes ni les permiten alcanzar una buena calidad de vida, ni su completa integración al mundo laboral (p. 70).

Aunado a lo anterior, Gutiérrez, et al. (2019), en su estudio realizado en Colombia, menciona la deserción laboral es un producto de un sistema que no ha sido capaz de articular el perfil y la vocación; por lo que desde dicho documento se propone que "la estructura académica de la formación superior debe responder precisamente a distintos frentes, entre los que se cuenta la oferta de programas." (párr. 5). Además de esta oferta de programas, se menciona el papel de las capacitaciones realizadas por los empleadores en los diversos puestos laborales que requieren según las diversas transformaciones. Esto es, de forma negativa, expresado en la realidad por Salas (2004) que "en definitiva, las empresas pueden estar más interesadas en "hacer» que en «comprar» empleados productivos" (p. 2).

Ahora bien, profundizando un poco en el comportamiento de la situación laboral, Pérez (2005) afirma que dentro del campo laboral se ha visto cierta inestabilidad, que está sujeta a cambios en este campo y que "toda polémica sobre la inestabilidad laboral, no puede desprenderse de la referencia a una construcción ideal acerca de lo que debe de ser el trabajo en la sociedad contemporánea" (p. 881). En Colombia, por ir al caso particular que nos compete ahora, para el 2019 "el total de ocupados se redujo en 193 mil personas con respecto al mismo periodo del año anterior, lo cual implica una disminución anual de 0,9\%" (Fedesarrollo, 2020, p. 3) y una tasa de desempleo nacional del $9.3 \%$ (debemos tener en cuenta también que estas cifras depende de la tasa global de participación); pero, si bien esto es cierto, en el mismo documento se evidencia, para el mismo periodo de tiempo en Colombia, "la generación de empleo estuvo impulsada por el crecimiento en las actividades de construcción y servicios sociales y personales" (Fedesarrollo, 2020, p. 3).

De esta forma, teniendo en cuenta el contexto actual, pasamos a abordar lo que es el campo de actuación social $y$, más específicamente del programa de Trabajo Social. Respecto a lo primero, debemos citar la necesidad que señala Palacios y Roca (2017), de "Incorporar acciones de pensamiento y de producción referidos a las formas como proceden los científicos sociales y de dar un valor central a los conocimientos previos y a los intereses de los estudiantes (p. 1), ligando así, estas necesidades con tema central de la presente investigación: las expectativas laborales de los estudiantes de educación superior. Los mismos autores, citando a Cajiao (1989), describen el objetivo de la enseñanza en ciencias sociales y su importancia - objetivo que permite tener en cuenta, desde lo que se espera en el marco de la realidad social, de la facultad de ciencias sociales y por ende la demanda del campo laboral de dichos profesionales, como agentes conocedores y transformadores de la realidad social (Farías, 2016) -, a saber:

[...]el objetivo de la enseñanza de las ciencias sociales es lograr que la persona sea capaz de hacer una reflexión comprensiva acerca de su acontecer individual, inmerso en su entorno social, resultado de un proceso histórico a lo largo del cual los grupos humanos han construido formas de organizarse, relacionarse, ubicarse, amarse, defenderse, expresarse, producir e interpretar la realidad; proceso que tiene sentido en cuanto permite intervenir como persona y como colectividad en la modificación de las condiciones 
de la vida heredadas, con el

fin de ser protagonistas en

la construcción de nuevos

modelos sociales y culturales

(p. 4).

Ahora bien, dentro la intervención desde el Trabajo Social, debemos traer aquí lo que argumenta Iturrieta (2012), para quien el Trabajo Social se caracteriza por su inespecificidad como profesión; la autora, basada en sus entrevistas a 56 trabajadores sociales chilenos, demarca que, actualmente, en el ejercicio laboral no hay delimitación disciplinar, ya que "Todos los trabajadores sociales están preparados para hacer de todo, no como otros profesionales que tienen como unárea específica" (p. 166), además, el ejercicio profesional de los Trabajadores Sociales es "tan amplio como problemas hay" (p. 166). Ahora bien, la autora identifica que el Trabajo Social ha perdido campos laborales de actuación a razón de "la escasa producción de conocimientos, sumada a la sensación de menoscabo profesional y a la vaguedad en el ejercicio profesional" (p. 168), no sin dejar de aclarar que sí hay especificidades el Trabajo Social: "la especificidad profesional estaría dada por el trabajo en el terreno; la aplicación de determinados modelos de intervención; conocimientos sobre familias; la elaboración de informes sociales y la asistencia social." (p. 168).

A nivel internacional encontramos estudios como el de Santana (2019), quien hace un abordaje de las expectativas que tienen los estudiantes de último año de Trabajo Social en España sobre su campo de actuación como la violencia intrafamiliar y de género, casos bastante presentes para el autor en la realidad social, y a partir de los cuales genera resultados en torno a lo que creían los estudiantes de Trabajo Social que sería el trabajo con víctimas de la violencia, que además de un mencionado miedo a sus afectaciones físicas y psicológicas:

\begin{abstract}
Por un lado, creen que necesitarán competencias específicas para ayudar a las mujeres a conseguir el empoderamiento y abandonar este tipo de relaciones. Por otro lado, se les plantearán diversos dilemas éticos cuando se enfrenten a situaciones donde hay menores involucrados y deban poner en marcha mecanismos protectores, igualmente cuando las mujeres se exponen a una revictimización que puede amenazar su integridad física, o cuando tengan que tratar con los agresores (p. 211).
\end{abstract}

Pero, siendo un poco más genéricos abordaremos algunas áreas de intervención intercultural y comunitaria desde el campo del Trabajo Social como lo son: tercera edad infancia - adolescencia, junto a drogodependencia, personas con discapacidad, adultos, salud mental, atención sociocomunitaria e inmigrantes. Áreas que se obtienen de un estudio realizado por López, et al. (2014), desde las concepciones de los estudiantes de educación y Trabajo Social.

Por su parte, en México, Arrollo, et al. (2012), refiere las áreas de intervención en Trabajo Social divididas en tres: área de salud, educativa y laboral. Dentro del área de la salud, el profesional busca "identificar e intervenir en los factores sociales que inciden en las condiciones de salud de la población derechohabiente" ( $p$. 27); el área educativa se refiere a la protección de derechos de los niños dentro de las instituciones educativas, seguimiento de la deserción escolar, al igual que de la asistencia de los estudiantes, gestión y registro de los recursos educativos necesarios, procesos inclusivos en la educación, orienta y lleva un registro de la situación "social 
y familiar de los alumnos" (p. 28), entre otras; por último, el área familiar "tiene como objetivo la mejora de alguna de las condiciones de sus miembros. Esta mejora implica, sin duda, la movilización de elementos personales y relaciónales 1: sentimientos, actitudes, comportamientos, y asimismo movilización y utilización de elementos externos" (p. 30).

Los trabajadores sociales tienen de esta forma una serie de salidas profesionales, sintetizadas por Martínez (2016), en: a.) servicios sociales comunitarios, que se configuran como puerta de entrada para los diferentes recursos del propio sistema público de servicios sociales y para la orientación y derivación, en su caso, hacia otras prestaciones y recursos de otros sistemas de protección social."; b.) "servicios sociales especializados, que dirigen su atención a personas que demandan un enfoque diferencial; c.) salud, como aquellas salidas profesionales en los centros de salud o servicios de atención primaria de salud, en servicios de atención sociosanitaria domiciliaria a enfermos con patologías crónicas y terminales, Hospitales generales. Maternales. Hospitales infantiles en centros sociosanitarios: atención sociosanitaria a pacientes crónicos y terminales; unidades de cuidados paliativos, entre otros; d.) educación y formación, donde la autora presenta salidas profesionales como la educación reglada, educación no reglada y educación en Trabajo Social; e.) justicia, donde el trabajador se desenvolverá con la justicia juvenil, los juzgados y las instituciones penitenciarias; e.) sector privado, donde se dará atención y prestaciones sociales a los trabajadores de determinadas empresas que cuentan con este servicio; f.) vivienda, donde generará acciones en pro de programas de promoción, estudio y adjudicación de viviendas de protección social; dinamización comunitaria de barrios de nueva construcción o en proceso de remodelación; planificación urbana; g.) tercer sector, donde trabajará con organizaciones y entidades $\sin$ ánimo de lucro que trabajan contra la exclusión social con programas preventivos, asistenciales, formativos, de inserción social y laboral a nivel local, desarrollando en muchos casos también programas de cooperación internacional. y, h.) Nuevos ámbitos de intervención, donde la autora lista ámbitos como planificación estratégica de servicios de bienestar, el análisis y desarrollo organizacional, asesoramiento en la dirección de políticas de bienestar social, marketing social, couseling, entre otros.

En un sentido más general nos podemos remitir a los argumentos de Franceschi (1996), quien llamó las previsiones que tiene la profesión de Trabajo Social sobre lo que se puede conseguir desde esta y que genera interrogantes en torno a "derivar las posibilidades de acción profesional a partir del análisis de la interacción de agentes sociales en un ámbito concreto, es decir, en una sociedad y en un tiempo determinado." (p. 2), el autor menciona que actualmente "es distinta la exigencia del perfil profesional, que demanda mayores conocimientos (teóricos, metodológicos e instrumentales) y capacidad para aplicarlos en la resolución de problemas socioeconómicos, socios ambientales, socioculturales concretos" (p. 4) donde el trabajador social tiene sus rasgos profesionales específicos, según el autor:

En los diversos campos
debemos proyectar la
aplicación de las habilidades y
destrezas que nos caracterizan
como profesión, tales como la
relación teórico- práctica y la
capacidad de comunicación
con las personas, frente a
las situaciones que requieren
respuestas profesionales.
En otras palabras, la
especialización en uno o varios
campos de acción determinados
(salud pública, microempresas,
niños y niñas trabajadoras de


y en la calle, entre otros), nos permitirán afirmarnos como profesionales de las ciencias sociales, con una identidad propia, por la pertinencia y relevancia de nuestros aportes (p.5).

\section{METODOLOGÍA}

Esta investigación se ajusta a las características del enfoque cuantitativo a nivel descriptivo transversal, con esto se pretende explorar las expectativas laborales que poseía una muestra no probabilística de profesionales en formación de un programa de Trabajo Social de la Facultad de Educación de una universidad pública del nororiente colombiano. De la revisión de antecedentes en el tema de interés, se creó un documento ad hoc el cual rescata de diversos aspectos hasta construir una encuesta compuesta de 41 ítems distribuidos de forma no equitativa en cinco categorías de observación: perfil demográfico, características académicas, situaciones que los informantes valoran en el trabajo, aporte del futuro profesional al ejercicio de su profesión y las características que en opinión de ellos debería poseer el trabajo ideal.

La primera versión de la encuesta fue revisada en detalle por el grupo de investigadores quienes actúan como juicio de expertos, para realizar ajustes en cuanto redacción y escalas de respuesta pertinentes. Es en ese sentido en dónde se concluye que las dos primeras categorías deberían ofrecen opciones de respuesta cerrada. Entre tanto las tres categorías siguientes hacen uso de una escala Likert con cinco niveles de valoración. A partir de estos ítems valorados en escala Likert, se pudo calcular el estadístico alfa de Cronbach el cual arrojó un valor de 0.938 , que en argumentos de George y Mallery (2003) se puede concluir que el cuestionario es fiable.
El instrumento se habilitó por tres semanas logrando la respuesta de 150 personas. Los datos fueron descargados en un archivo de Excel y posteriormente fueron exportados al software SPSS v25 para ser procesados de forma descriptiva con el fin de generar frecuencias, porcentajes $y / 0$ diversas representaciones gráficas que aportaran a la caracterización de las expectativas laborales que poseen los profesionales en formación con respecto a su futuro laboral.

\section{CARACTERIZACIÓN DE LA MUESTRA}

En cuanto a las características demográficas y académicas de los informantes se resalta que:

a) En cuanto al género, el $76.7 \%$ son mujeres.

b) Se identificó que 18 años fue la edad mínima y 23 la edad máxima, con una media de 20.4 y desviación estándar de 1.28 años, con distribución asimétrica positiva $(0.102)$ y platicúrtica (curtosis de -0.474).

c) En lo que respecta al estado civil, se determinó que el $96.7 \%$ son solteros y el porcentaje restante tiene una relación formal.

d) Al explorar sobre la composición familiar de los informantes, se determinó que el $93.3 \%$ de ellos viven con sus padres y hermanos, el $3.3 \%$ viven con otro familiar y el $3.3 \%$ restante con su pareja. Se destaca que aquellos que son casados, a la fecha no tienen hijos.

e) Se contó en la muestra con la participación de estudiantes de cinco programas diferentes de la Facultad de Educación, que fueron: Comunicación Social con el $19.3 \%$, Derecho con el $20.7 \%$, Licenciatura en Matemáticas con el 23.3\%, Licenciatura en Educación Infantil con el $16.1 \%$ y Trabajo Social con el $20.6 \%$.

f) Se contó con estudiantes que cursaban entre el tercero y el noveno semestre académico de sus propuestas curriculares, concentrando el 
$45 \%$ de los informantes en los semestres cuarto, quinto y sexto.

\section{RESULTADOS}

Como se mencionó en la sección anterior, se generó un conjunto de 34 ítems distribuidos en tres categorías, las cuales permiten explorar los aspectos que son importantes para los estudiantes en su futuro laboral, así como la percepción de impacto social del ejercicio de su profesión.

\section{CARACTERÍSTICAS QUE MEJOR VALORAN LOS ESTUDIANTES DE UN TRABAJO}

Al analizar la opinión de los encuestados en lo que respecta los aspectos que considera importantes en un trabajo, se pudo determinar

Al analizar en detalle cada uno de los aspectos o características referidas con el fin de que los profesionales en formación valorarán el nivel de importancia dentro de la consideración de un trabajo; se identificó que el salario, el ambiente de trabajo, la posibilidad de continuar cualificándose al ejercer su labor, la proyección que en promedio el $91 \%$ de ellos reconocen lo transcendental de las características mencionadas en esta categoría.

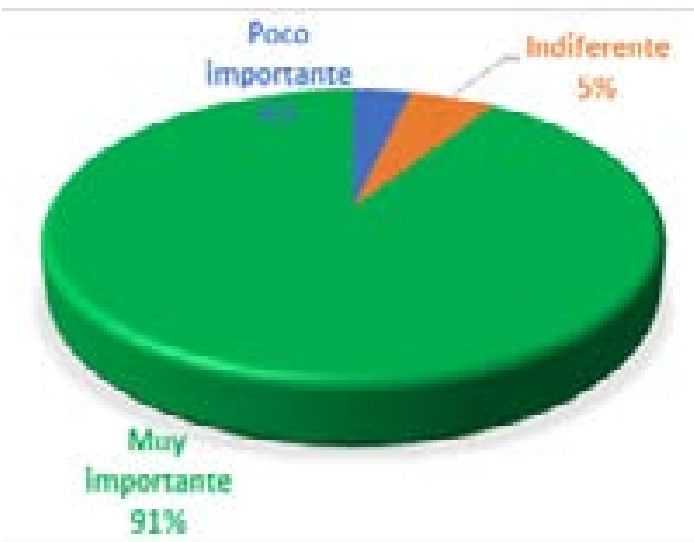

Figura 1. Nivel de importancia promedio que le da el informante a los aspectos referidos con las características que valora de un trabajo

social de su trabajo y el equilibrio que puede tener con su vida personal o familiar, resultaron ser lo más relevante a la hora de considerar un trabajo. Así mismo, se identificó que la cercanía del trabajo con su lugar de residencia, resultó ser una característica poco relevante en algunos casos.

Tabla 1. Características que valora como importantes en un trabajo en opinión de los informantes

\begin{tabular}{lccc}
\hline \multirow{2}{*}{ Característica } & \multicolumn{3}{c}{ Nivel de relevancia } \\
\cline { 2 - 4 } & Poco importante & Indiferente & Muy importante \\
\hline Salario & $0.0 \%$ & $3.3 \%$ & $96.7 \%$ \\
\hline Estabilidad en el puesto de trabajo & $3.3 \%$ & $3.3 \%$ & $93.4 \%$ \\
\hline Prestigio de la empresa & $6.7 \%$ & $10.0 \%$ & $83.3 \%$ \\
\hline Ambiente de trabajo & $0.0 \%$ & $3.3 \%$ & $96.7 \%$ \\
\hline Posibilidad de desarrollo profesional & $0.0 \%$ & $6.7 \%$ & $93.3 \%$ \\
\hline Horario de trabajo & $3.3 \%$ & $3.3 \%$ & $93.4 \%$ \\
\hline Cercanía con su vivienda & $13.3 \%$ & $26.7 \%$ & $60.0 \%$ \\
\hline Posibilidad de aprendizaje y formación & $3.3 \%$ & $0.0 \%$ & $96.7 \%$ \\
\hline $\begin{array}{l}\text { Posibilidad de ascender dentro de la } \\
\text { estructura jerárquica de la empresa }\end{array}$ & $3.3 \%$ & $3.3 \%$ & $93.4 \%$ \\
\hline Proyección social del trabajo realizado & $3.3 \%$ & $0.0 \%$ & $96.7 \%$ \\
\hline $\begin{array}{l}\text { Equilibrio entre la vida personal } \\
\text { familiar }\end{array}$ & $3.3 \%$ & $0.0 \%$ & $96.7 \%$ \\
\hline \multicolumn{1}{c}{ Porcentaje promedio } & $\mathbf{3 . 6 \%}$ & $\mathbf{5 . 4 \%}$ & $\mathbf{9 0 . 9 \%}$ \\
\hline
\end{tabular}




\section{APORTE DEL EJERCICIO LABORAL AL DESARROLLO DE SU PROFESIÓN}

En opinión de los encuestados, en promedio el $91 \%$ reconocen y manifiestan la importancia de algunas acciones que, desde su futuro desempeño profesional, aportarían al buen ejercicio de la profesión y contribuiría a la aplicación de altas competencias como lo muestra la gráfica subsiguiente:

Cuando se revisa detalladamente los aportes que cada encuestado se puede considerar que desde su futuro en ejercicio laboral algunos elementos importantes se relacionan con el liderazgo reflejado en la capacidad de orientar a otras personas y en la prestación de un servicio de calidad; seguido de la actualización

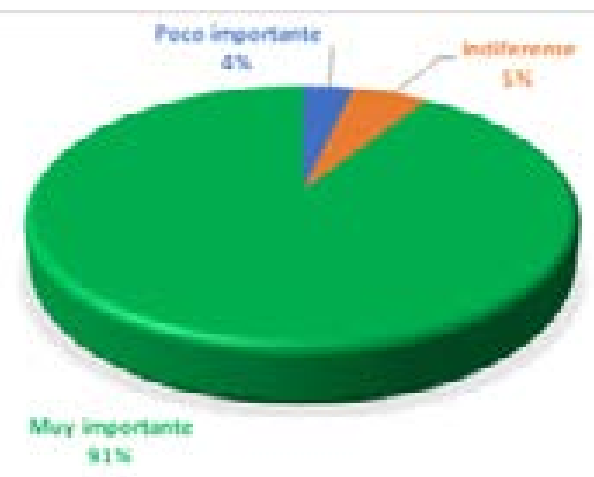

Figura 2. Percepción sobre el aporte que desde su trabajo puede hacer con la profesión

permanente y su autoestima que se refleja en el sentirse útil y el alcanzar el éxito profesional. Estas características se complementan con una postura altruista, dado que un buen porcentaje de ellos aseguran que el reconocimiento social y la admiración no es su principal motor.

Tabla 2. Aporte del ejercicio al desarrollo de la profesión

\begin{tabular}{lccc}
\hline \multirow{2}{*}{ Característica } & \multicolumn{3}{c}{ Nivel } \\
\cline { 2 - 4 } & Poco importante & Indiferente & Muy importante \\
\hline Ser capaz de orientar a los demás & $0.0 \%$ & $3.3 \%$ & $96.7 \%$ \\
\hline Prestar un servicio a los demás & $0.0 \%$ & $3.3 \%$ & $96.7 \%$ \\
\hline $\begin{array}{l}\text { Tener acceso a buenas relaciones } \\
\text { sociales }\end{array}$ & $0.0 \%$ & $6.7 \%$ & $93.3 \%$ \\
\hline Superación permanente & $0.0 \%$ & $10.0 \%$ & $90.0 \%$ \\
\hline Aprendizaje constante & $0.0 \%$ & $3.3 \%$ & $96.7 \%$ \\
\hline Integración a equipos de trabajo & $0.0 \%$ & $10.0 \%$ & $90.0 \%$ \\
\hline Sentirse aceptado por los demás & $3.3 \%$ & $10.0 \%$ & $86.6 \%$ \\
\hline Obtener prestigio en lo que realiza & $0.0 \%$ & $10.0 \%$ & $90.0 \%$ \\
\hline Recibir admiración y reconocimiento & $6.7 \%$ & $13.3 \%$ & $80.0 \%$ \\
\hline Autonomía en mi trabajo & $0.0 \%$ & $10.0 \%$ & $90.0 \%$ \\
\hline Alcanzar el éxito profesional & $0.0 \%$ & $3.3 \%$ & $96.7 \%$ \\
\hline Conocer nuevas experiencias laborales & $0.0 \%$ & $6.7 \%$ & $93.3 \%$ \\
\hline Sentirse útil & $0.0 \%$ & $3.3 \%$ & $96.7 \%$ \\
\hline Innovación permanente & $0.0 \%$ & $6.7 \%$ & $93.3 \%$ \\
\hline \multicolumn{1}{c}{ Porcentaje promedio } & $\mathbf{0 . 7 \%}$ & $\mathbf{7 . 1 \%}$ & $\mathbf{9 2 . 1 \%}$ \\
\hline
\end{tabular}




\section{PERFIL DEL TRABAJO IDEAL}

En opinión de los encuestados, en promedio el $72 \%$ de ellos reconocen como relevantes algunas de las características que se mencionan en esta categoría y que consideran serían valiosa en el trabajo que esperarían ubicarse una vez se gradúen de su proceso de formación profesional. En esta última categoría se resalta que aproximadamente dos de cada diez encuestados manifiestan que les resulta indiferente los aspectos mencionados, luego se podría pensar que lo importante sería el

Al indagar entre los encuestados sobre los elementos más importantes que debería ofrecerles un trabajo ideal está como principal elemento diferenciador que el trabajo les permita la oportunidad de seguirse preparando para ser cada día más y más competentes. Le siguen en conseguir trabajo independientemente de las condiciones en que se genere el mismo.

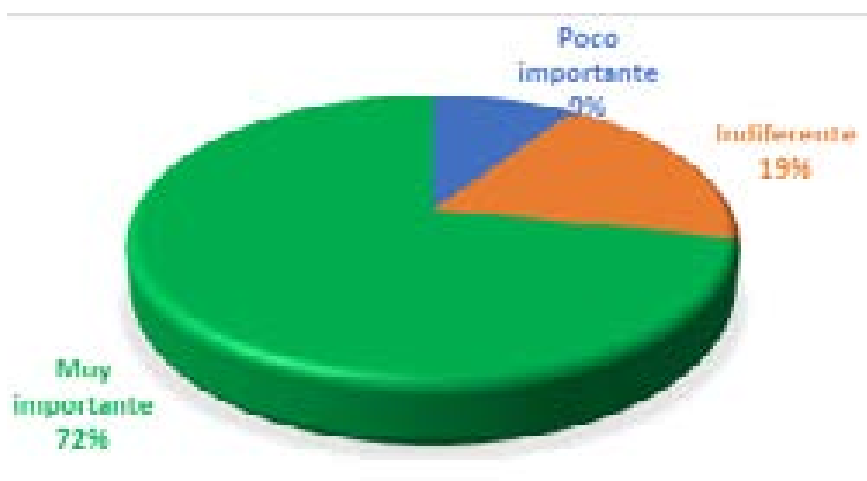

Figura 3. Percepción sobre las características del trabajo ideal para cada encuestado

orden de importancia, la realización de trabajo en equipo, pero preferiblemente asumiendo ellos el rol de líder de dichos equipos; y finalmente, de las condiciones laborales más rechazas se encuentran el sueldo bajo y la poca estabilidad.

Tabla 3. Características del trabajo ideal en opinión de los encuestados

\begin{tabular}{|c|c|c|c|}
\hline \multirow{2}{*}{ Característica } & \multicolumn{3}{|c|}{ Nivel } \\
\hline & Poco importante & Indiferente & Muy importante \\
\hline $\begin{array}{l}\text { Que el trabajo a realizar se alcance con } \\
\text { otros empleados }\end{array}$ & $6.7 \%$ & $3.3 \%$ & $90.0 \%$ \\
\hline Poder avanzar en su vida profesional & $0.0 \%$ & $3.3 \%$ & $96.7 \%$ \\
\hline $\begin{array}{l}\text { Tener tiempo libre, aunque el sueldo no } \\
\text { sea bueno }\end{array}$ & $13.3 \%$ & $36.7 \%$ & $50.0 \%$ \\
\hline $\begin{array}{l}\text { Poder ejercer la profesión a pesar de } \\
\text { que el sueldo sea bajo }\end{array}$ & $30.0 \%$ & $30.0 \%$ & $40.0 \%$ \\
\hline $\begin{array}{l}\text { Tener responsabilidades y dar cuenta } \\
\text { de ellas }\end{array}$ & $6.7 \%$ & $6.7 \%$ & $86.6 \%$ \\
\hline Que me permita seguir preparándome & $0.0 \%$ & $0.0 \%$ & $100.0 \%$ \\
\hline Que sea creativo con poca estabilidad & $16.6 \%$ & $43.3 \%$ & $40.0 \%$ \\
\hline Que me permita viajar frecuentemente & $10.0 \%$ & $36.7 \%$ & $53.3 \%$ \\
\hline En el que coordine equipos de trabajo & $0.0 \%$ & $10.0 \%$ & $90.0 \%$ \\
\hline Porcentaje promedio & $9.3 \%$ & $18.9 \%$ & $71.8 \%$ \\
\hline
\end{tabular}




\section{CONCLUSIONES Y DISCUSIÓN}

Los resultados del estudio demuestran que existen elementos importantes que motivan a los Trabajadores Sociales en formación con respecto a sus expectativas laborales, las cuales se relacionan con: el ambiente de trabajo, la posibilidad de continuar cualificándose al ejercer su labor, la proyección social de su trabajo y el equilibrio que puede tener con su vida personal o familiar.

Las expectativas laborales de los encuestados según el estudio, se pueden relacionar con elementos altruistas, ya que sus respuestas tienen una tendencia a procurar el bien de las personas de manera desinteresada, incluso a costa del interés propio. Estos resultados se pueden interpretar desde los resultados de los estudios como los de García-Quintero y Villamizar-Suárez (2017) o de Mayoral y Laca (2013) quienes afirman que los trabajadores en el campo social tienen actitudes altruistas debido a un desinteresado sentimiento de bienestar social y un clima emocional de empatía que surge de su formación académica.

Por otra parte, en cuanto a los ideales del Trabajador Social según los datos analizados, se puede rescatar que el trabajo en equipo y el liderazgo, son las proyecciones más recurrentes de los encuestados. En este sentido estudios como los de Maestre y Simón (2000) y Kisnerman (1998) afirman que el trabajo en equipo en cualquier profesión se caracteriza por la definición de objetivos, la delimitación clara y estructurada de las funciones de cada grupo profesional, la participación de todos sus componentes en la toma de decisiones, la comunicación y las actitudes activas y facilitadoras del trabajo en equipo

Por último es importante señalar que algunos de los temores de los profesionales en formación con respecto al futuro de su profesión son en su orden la baja remuneración económica y la inestabilidad laboral. Al respecto Ruoti (2017) afirma que una de las mayores preocupaciones de los profesionales en el campo social es la alta responsabilidad que tienen con las problemáticas sociales y la poca valoración económica y laboral que este rol exige.

\section{REFERENCIAS BIBLIOGRÁFICAS}

Arroyo, E., García, R., Pineda, G., Velázquez, K., Peña, I., Hidalgo, A., Vergara, E. (2012). Funciones laborales del trabajador social. Universidad Veracruzana. https://www.uv.mx/personal/eperry/ files/2011/05/evidencias-invest.cuantitativa-funciones-del-trabajadorsocial-en-el-ambito-laboral.pdf

Cabezas, B., Giner Martínez, A., Méndez, G., Podlesnyk Mikhayela, M., \& Sansa Aizcorbe, A. (2013). Expectativas, motivación y satisfacción de los estudiantes de GAP. Diposit Digital de la Universitat de Barcelona 1- 61. https:// core.ac.uk/reader/2031893

Farías, F. (2016). Estudio Exploratorio de los/as profesionales de las ciencias sociales en las comunas del gran concepción, Chile. TS Cuadernos de Trabajo Social, (5), 1-13. file:///C:/Users/usuario/ Downloads/54-Texto\%20del\%20 art\%C3\%ADculo-103-1-10-20160709. pdf

Fedesarrollo, Centro de Investigación Económica y Social. (2020). Informe Mensual Del Mercado Laboral. Gobierno colombiano. https://www.bibguru.com/es/g/cita-apainforme/

Franceschi, H. (1996). Expectativas del trabajo social en el ámbito profesional dentro del contexto neoliberal. Boletín Electrónico Surá (5).1 - 6 http://www.ts.ucr.ac.cr/ binarios/sura/sura-0005.pdf 
García-Quintero, C., \& Villamizar Suárez, G. (2017). Análisis fenomenológico de la conciencia del docente a partir de sus prácticas evaluativas. Revista Perspectivas, 2(2), 49-59. https://doi. org/10.22463/25909215.1313

Gutiérrez, J.A, Mondragón, V., \& Santacruz, L. (2019). Expectativas, necesidades y tendencias de la formación en educación superior en Colombia en pregrado y posgrado: entre la deserción-perfil y vocación profesional. Universidad \& Empresa, 21(37), 313-345. https://doi. org/10.12804/revistas.urosario.edu.co/ empresa/a.6619

George, D., \& Mallery, P. (2003). Reliability analysis. SPSS for Windows, step by step: a simple guide and reference, 14th edition. Boston: Allyn \& Bacon, 222-232.

Kisnerman, N. (1998). Pensar el trabajo social. Sites: Lumen.

Maestre, M. V., \& Simón, S. C. (2000). Opiniones y expectativas de los profesionales de atención primaria sobre el trabajo en equipo. Atención primaria, 26(5), 309313.

Martínez, S. (20 de diciembre del 2016). Ámbitos de intervención y salidas profesionales trabajo social. Revista Digital INESEM, Educación y Sociedad. https:// revistadigital.inesem.es/educacionsociedad/salidas-profesionales-entrabajo-social/

López, E.,Vázquez, E., Fernández, E. (2014). Análisis de la percepción de los estudiantes sobre las áreas de intervención del futuro educador y trabajador social a través de una didáctica digital con mapas conceptuales multimedia. Revista de educación a distancia, (41), 1 - 17. http://hdl.handle. $\underline{\text { net/10201/46548 }}$

Mayoral Sánchez, Edwin G. y Laca Arocena, Francisco A. (2013). Altruismo y bienestar social en la explicación de voluntariado en estudiantes mexicanos de bachillerato y licenciatura. Psicología Iberoamericana, 21 (2), 8595. https://www.redalyc.org/articulo. oa? $\mathrm{id}=133930525009$

Palacios, P., \& Roca, E. (2017). el aprendizaje de las ciencias sociales desde el entorno: Las percepciones de futuros maestros en el geoforo iberoamericano de educación. Biblio3W Revista Bibliográfica de Geografía y Ciencias Sociales. http://www.ub.edu/geocrit/ $\underline{b 3 w-1204 . p d f}$

Paz, F., Betanzos, N., y Uribe, N. (2014). Expectativas laborales y empleabilidad en enfermería y psicología. Aquichan, 14(1), 67-78. file:///C:/Users/usuario/ Downloads/Dialnet-ExpectativasLab oralesYEmpleabilidadEnEnfermeriaY Ps-4955976.pdf

Pérez, M. (2005). Trabajo y familia: Posiciones optimistas y pesimistas en torno a la inestabilidad laboral. Estudios Sociológicos, 23(69), 879-892. http:// www.jstor.org/stable/40421011

Pinazo, D., Carrero, V. (2001). El Desajuste De Expectativas Laborales y La Permanencia En EI Puesto De Trabajo. Revista Española de Motivación y Emoción, 3(2). 99-106. http://reme.uji. es/articulos/apinad6121102100/texto. $\underline{\mathrm{html}}$

Real Academia Española. (s.f.). Expectativa. En Diccionario de la lengua española. Recuperado en 04 de mayo del 2021. https://dle.rae.es/expectativa 
Ruoti, M. (2017). Perfil ocupacional y estrategias de actuación profesional de trabajadores sociales en el contexto paraguayo. Kera Yvoty: Reflexiones Sobre La cuestión Social, 2, 44-56. Recuperado de https:// revistascientificas.una.py/index.php/ kerayvoty/article/view/452

Santana, J. D. (2019). Expectativas de los estudiantes de Trabajo Social sobre su desempeño profesional con víctimas de violencia familiar y de género: un estudio cualitativo. Trabajo Social, 21(1), 195-217. https://doi.org/10.15446/ ts.v21n1.69983

Iturrieta, S. (2012). Desafíos del trabajo social en un campo laboral con límites profesionales difusos. $R$. Katál., Florianópolis, 15(2), p. 163-172. https:// www.scielo.br/pdf/rk/v15n2/01.pdf

Salas, M. (2004). La Relación EducaciónEconomía: un estudio del desajuste educativo de los titulados universitarios. Revista de Educación, (334). 259278. http://www.educacionyfp.gob. es/dam/jcr:6df11df4-5d54-4a3b-ab8f3ccdea4c6ca2/re33416-pdf.pdf 